\title{
Deep Unity of Classic and Quantum Physics at the Space Thermostat Presence with Technical Applications
}

\author{
M. Ja. Ivanov ${ }^{1}$, V. K. Mamaev ${ }^{1}$, Guanghua Zheng² \\ ${ }^{1}$ Gas Turbine Department, Central Institute of Aviation Motors, Moscow, Russia \\ ${ }^{2}$ Northwestern Polytechnic University, Xian, China \\ Email: ivanov@ciam.ru,mamaev@ciam.ru, zhengguanghua@nwpu.edu.cn
}

How to cite this paper: Ivanov, M.J., Mamaev, V.K. and Zheng, G.H. (2017) Deep Unity of Classic and Quantum Physics at the Space Thermostat Presence with Technical Applications. Journal of Applied Mathematics and Physics, 5, 801-812. https://doi.org/10.4236/jamp.2017.54069

Received: October 25, 2016

Accepted: April 9, 2017

Published: April 12, 2017

\begin{abstract}
The paper demonstrates deep unity of classic and quantum physics at the space thermostat (ST) presence, which fulfilled all space by the temperature $T_{0}$ $=2.73 \mathrm{~K}$. The ST presents itself the Cosmic Microwave Background (CMB). From the main quantum position we consider the ST/CMB as the wave function carrier ("quantum background"). The paper is devoted to ST/CMB medium the classic conservation laws of mass, momentum and energy. We show the soliton like solutions of our classic model correspond to Schrodinger's quantum solutions, demonstrate the atom hydrogen specter and other quantum peculiarities. The paper contains typical technical examples classic/ quantum simulation at the ST presence.
\end{abstract}

\section{Keywords}

Space Thermostat, Conservation Laws, Schrodinger's Solutions, Technical Applications

\section{Introduction}

Some achievements in experimental physics and astrophysics during of a few last decades show that it would be useful additionally to study important background theoretical aspects for classic and quantum physics. First of all among such experimental achievements we name the registration of the Cosmic Microwave Background (CMB) with the finite temperature $T_{0}=2.73 \mathrm{~K}$ [1]-[5]. The second significant achievement is the discovery of Dark Matter/Energy (DME) [6] [7], which is also called "physical vacuum". Now we know that $96 \%$ whole substance in our Universe consists of DME. The baryonic substance accounts to only near $4 \%$. 
The paper is devoted to CMB as the Space Thermostat (ST) medium with the known (no zero) temperature and finite mass particles and presents the classic conservation laws of mass, momentum and energy [8]. We show the soliton like solutions of our classic model correspond to Schrodinger's quantum solutions [9], demonstrate the atom hydrogen specter and other quantum peculiarities.

From the main theoretical position we consider the ST/CMB as the carrier of quantum wave function and quantum phase trajectories ("quantum background"). At present time it seems to be indeed that quantum mechanics cannot be formulated by simply considering a statistical approximation from a classical-like deterministic theory.

Alternatively, it can be also interpreted as the evolution of a quantum flow in a hydrodynamic form of quantum mechanics [10]. In 1952 Bohm proposed a physical hidden-variable model [11]-[13] which reproduced the predictions of the standard quantum theory without violating any of its postulates. The main goal of this work consists of helping to develop a common united appropriate classic and quantum physics similar Bohmian mechanics at the ST/CMB presence.

\section{The Space Thermostat: Properties and Parameters}

In physics a thermostat is called a greater thermodynamic system, the number of particles which far exceeds the number of particles in a studied system with her in thermal contact [14]-[16]. In our case as such thermostat (the large thermodynamic system) we take the real Cosmic Microwave Background (CMB) of our Universe and dark matter/energy (DME) medium with certain massive particles (see below), which are holders and carriers of thermal radiation (and, in particular, CMB). Any smaller size natural or technical system we believe in the thermal contact with the specified Space Thermostat (ST). The considered ST essentially differs from ordinary Gibbs' thermostat [14], in which studied systems are in thermal contact by $T \gg 2.73 \mathrm{~K}$ and have the same temperature.

Our ST is radiate compressible medium and bearer of quantum wave function and quantum phase trajectories ("quantum background"). Following the [8] we believe that the thermal radiation (and, in particular, CMB) behaves like an ideal gas with adiabatic factor $\gamma=4 / 3$ and is synonymous in this sense with photon gas. Coming back from some fundamental ideas (first of all, from recommendations by M. Planck, A. Einstein and L. de Broglie) allows us to indicate a correlation linking energy $E$ with mass value $m$, frequency $v$ and temperature $T$.

$$
E=m c^{2}=h v=k T,
$$

where $c$. the light velocity; $h$ and $k$. the Planck and the Boltzmann constants. The last equality in (1) is the law of evenly distributed energy on freedom degrees. Also Equation (1) follows from Planck's distribution in vicinities of maximum radiation density of an absolutely black body and presents itself Wien's displacement law. The relation (1) allows us to define also the vacuum particle mass, when $T \neq 0$. The presence of these nonzero mass particles in physical va- 
cuum was specified in [8] and it was identified with massive particles of DM named Hidden Mass Boson (HMB). The same way we propose simulation for the Dark Energy (DE) [8]. To be short, we change the virtual Planck resonators in his derivation of the famous formula for absolutely black body radiation density by real (massive) particles with $m=k T / c^{2}$ (following from the relations (1)). Also the possibility of radiation (including of electromagnetic waves, similar the virtual Planck resonators) allows us to consider these real HMB particles as a classic Hertz's dipoles.

Considering the ST particle concentration $n$ and multiply (1) on $n$ we can write

$$
n \cdot m c^{2}=n \cdot k T
$$

and go to the typical ideal gas state equation

$$
p \approx \rho c^{2}=n k T .
$$

Here $\rho=n \cdot m-$ density, $p$ - pressure in ST medium. The relation (2) is one of the mathematical forms of Avogadro's law. Now we show that the recommendations by M. Planck, A. Einstein and L. de Broglie (1) may be considered as another form of Avogadro's law and the classic state equation for perfect gaseous medium (2). The relations (1) and (2) may be used for answers on intrigue question so to what comprises about $96 \%$ of content of the Universe (i.e., what and why over $70 \%$ of the mass-energy content of the Universe is in form of the unknown vacuum DE, over $20 \%$ of the mass is in the form of the mysterious DM).

Get through (1) and (2) refinement more accurate the value for the ST particle mass at $T_{0}=2.73 \mathrm{~K}$ and the perturbation velocity $c=2.998 \times 10^{8} \mathrm{~m} / \mathrm{s}$. We have obtained

$$
\begin{aligned}
& E=\frac{m v_{a v}^{2}}{2}=\frac{3}{2} k T_{0}=\frac{3}{2} \frac{R_{U}}{N_{A}} T_{0}=m \frac{3}{2} \frac{R_{U}}{m N_{A}} T_{0}=\frac{3}{2} \frac{m}{\gamma} \gamma R T_{0}=\frac{3}{2} \frac{m}{\gamma} c^{2} \\
& m=\gamma k T_{0} / c^{2}=5.6 \times 10^{-40} \mathrm{~kg}=3 \times 10^{-4} \mathrm{eV} .
\end{aligned}
$$

We calculate the gas constant $R$ and the specific heat capacity $c_{V}$ и $c_{p}$ by $\gamma=$ $4 / 3$

$$
R=\frac{k}{m}=0.25 \times 10^{17} \frac{\mathrm{J}}{\mathrm{kg} \mathrm{K}} ; c_{v}=0.75 \times 10^{17} \frac{\mathrm{J}}{\mathrm{kg} \mathrm{K}} ; c_{p}=R+c_{v}=1.0 \times 10^{17} \frac{\mathrm{J}}{\mathrm{kg} \mathrm{K}} .
$$

It should be stressed that the thermal radiation has the classic state equation

$$
p=\rho R T, p=(\gamma-1) \rho e, e=c_{v} T .
$$

The ST particles (3) are the sub-atomic (non-baryonic) material particles moving "almost" free in all directions at different velocities. One half of particles have positive charge and other half has negative identical in its value electrical charge [8]. Besides, pairs of the oppositely charged particles from the classic Hertz dipoles, which have with translational, rotary and oscillatory degree of freedom. We obtain a liner size of the dipole $I=7 \times 10^{-20} \mathrm{~m}$ and its charge $q=$ $10^{-28} \mathrm{C}$. The value of the electric dipole moment $p=q \cdot l=7 \times 10^{-48} \mathrm{C} \cdot \mathrm{m}$. In spite of its miniature size we consider that all known properties of electric dipoles are 
retained. Thus the medium as a whole is quasi-neutral; however there are socalled "collective" processes possible, such as a local concentration of positive and negative electrical charges.

\section{Noether's Theorem, Lagrangian and Hamiltonian Mechanics}

Looking at some consequences of the thermostat existence the question of the applicability of the Noether theorem in modern physics should be analyzed. The Noether theorem declaims that every differentiable symmetry of the action of a physical system has a corresponding conservation law [17]. By Noether's theorem the symmetries of translations in space and time get to the conservation laws of momentum and energy within this system, respectively. In the same time Noether's theorem takes place only for dissipative less isolate systems. In our case with ST and energy dissipation on its level we have no possibility to use Noether's theorem. The same situation takes place with the Lagrangian and the Hamiltonian mechanics.

There is used as the standard language of the particle physics to express in terms of Lagrangians [18]. To give the flavor of the general theorem, a version of the Noether theorem for continuous fields in four dimensional space - time has been given. The requirement of invariance of the Lagrangian for local gauge transformations is the original principle of all modern physics theories of a microcosm. The gauge transformation is rotation at arbitrary angles around the same axis in the Minkovsky 4-space. Here we have local time's arrow decline and rotation. It is one of the great differences between perspective new horizons and modern physics. We should include into account some principal limitations of the Lagrangian formalism and quantum field theories, which connect with time's arrow (real time's arrow has no possibility to decline at any side and twisting). The real physics has only the "one-way direction" arrow of time (without any rotation).

Further in our paper we analyze deep unity of classic and quantum physics at the presence of the external ST with energy changing (first of all, the energy dissipation on the level of ST). A few going forward we would like to emphasize that the growth of our system's entropy characterizes the amount of scattered (absorbed by ST) energy. Now we consider some items of Bohmian quantum mechanics.

\section{Some Bohmian Mechanics Background}

The wave function $\psi$ supplies the quantum system with dynamical information on each point of the associated configuration space at each time moment. Within quantum presentation of Bohmian mechanics [11]-[13] this information is encoded in its phase, as can be seen through the transformation relation

$$
\psi(r, t)=\rho^{1 / 2}(r, t) \mathrm{e}^{i S(r, t) / \hbar},
$$

where $\rho$ and $S$ are the probability density and phase of $\psi$, respectively, both being 
real valued quantities. This relation allows us to pass from the Schrödinger equation

$$
i \hbar \frac{\partial \psi}{\partial t}=\left(-\frac{\hbar^{2}}{2 m} \nabla^{2}+V\right) \psi,
$$

to the system of coupled equations [11]

$$
\begin{aligned}
& \frac{\partial \rho}{\partial t}+\nabla \cdot\left(\rho \frac{\nabla S}{m}\right)=0 \\
& \frac{\partial S}{\partial t}+\frac{(\nabla S)}{2 m}+V+Q=0 \\
& Q=-\frac{\hbar^{2}}{2 m} \frac{\nabla^{2} \rho^{1 / 2}}{\rho^{1 / 2}}=\frac{\hbar^{2}}{4 m}\left[\frac{1}{2}\left(\frac{\nabla \rho}{\rho}\right)^{2}-\frac{\nabla^{2} \rho}{\rho}\right]
\end{aligned}
$$

is the so-called quantum potential. Equation (7) is the continuity equation, which describes the ensemble dynamics, i.e., the motion of a swarm of trajectories initially distributed according to some $\rho_{0}$. Equations (8) and (9) govern the motion of individual particles, in particular, the quantum Hamilton-Jacobi Equation (8) accounts for the phase field evolution ruling the quantum particle dynamics through the equation of motion

$$
u=\nabla S / m .
$$

This relation indicates that one can define a local velocity field on each point of the system configuration space and, by integrating it in time, to obtain the corresponding trajectory.

In our study we get some next steps in Bohmian mechanics direction, in which the ST/CMB presents as the wave function carrier ("quantum background"). We consider $\rho$ as real ST medium density and $u$ as real ST medium velocity. By that we can write conventional equations of discontinuity and momentum (see the next section). Further the relations (1) and (2) allow introducing also pressure $p$ and temperature $T$ in our consideration of quantum Bohmian mechanics at the ST presence. Following classic mechanics we have possibility now to use additionally the equation of energy conservation.

\section{Some Classic Mechanics Background}

We present the common conservation laws for the case of the ST presence as the two components model of a gaseous and radiation (quantum) medium [8]. From the main quantum position we consider the ST/CMB as the quantum wave function carrier ("quantum background"). There are used the index $g$ for conventional gas and the index $f$ for radiation components of medium (for example, for densities $\rho_{g}$ and $\rho_{f}$ ). For the one velocity model the values of velocity components $u, v, w$ at the axis $x, y, z$ are the same for each medium components. The integral conservation laws are presented as [19] [20] for the volume $\omega(t)$ with the boundary $\gamma(t)$ 


$$
\begin{aligned}
& \frac{d}{d t} \iiint_{\omega(t)} \rho_{k} \mathrm{~d} \omega=\iiint_{\omega(t)} q_{k} \mathrm{~d} \omega, \\
& \frac{d}{d t} \iiint_{\omega(t)} \rho_{k} \boldsymbol{u} \mathrm{d} \omega=-\iint_{\gamma(t)} p_{k} \boldsymbol{n} \mathrm{d} \gamma+\iiint_{\omega(t)} \boldsymbol{r}_{k} \mathrm{~d} \omega, k=g, f \\
& \frac{d}{d t} \iiint_{\omega(t)} \rho_{k}\left(\frac{1}{2} q^{2}+\varepsilon_{k}\right) \mathrm{d} \omega=-\iint_{\gamma(t)} p_{k} \boldsymbol{u} \cdot \boldsymbol{n} \mathrm{d} \gamma+\iint_{\gamma(t)} K_{k} g r a d T_{k} \cdot \boldsymbol{n} \mathrm{d} \gamma+\iiint_{\omega(t)} L_{k} \mathrm{~d} \omega .
\end{aligned}
$$

Here $q^{2}$ - the square of the velocity vector $\boldsymbol{u}$ and

$$
L_{g}=C_{g f}\left(T_{f}-T_{g}\right)+Q_{g}^{\prime}, L_{f}=C_{g f}\left(T_{g}-T_{f}\right)+Q_{f}^{\prime} .
$$

Energy conservation laws are written for heat transfer gas and radiation components (the second terms in the right side of these equations, $K_{g}$ and $K_{f}$ correspondently thermo transfer coefficients for gas and radiation parts). The last terms in the right side of initial energy equations describe an energy exchange between gas and radiation parts (the space thermostat). The terms $Q_{g}^{\prime}$ and $Q_{f}^{\prime}$ are an additional energy souses.

\section{Some Examples of Quantum Hydrodynamic Analogy}

The achievement of a mutual systematic understanding of quantum and classical phenomena has been considered in [10]-[13]. In these papers, the standard quantum mechanics is derived as the deterministic limit of the stochastic hydrodynamic analogy. Now there is considered deep coordination of classic and quantum solutions on the example of a hydrogen atom specter and screening spaces of elementary particles.

At first we analyze a hydrogen atom specter (in particular, the Ballmer series, Figure 1) on the base of the system (11). The main steps of classic solution realization for a hydrogen atom specter are presented in [8] [21]. We also give

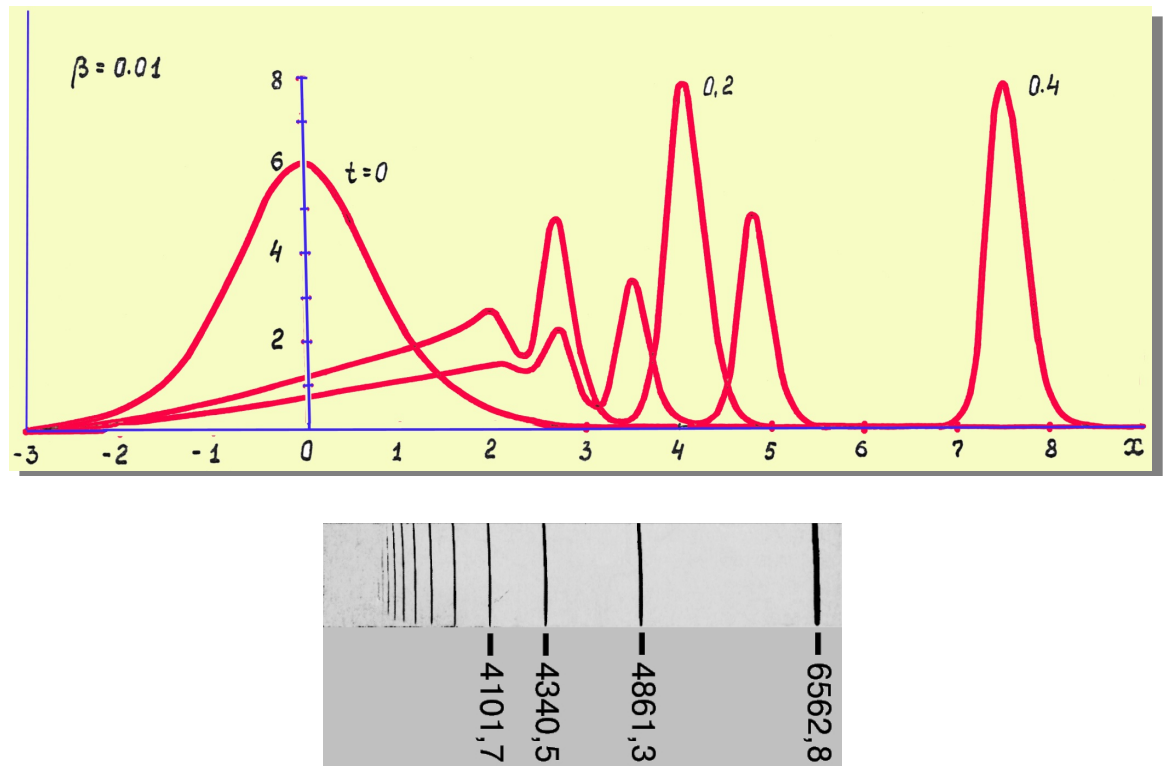

Figure 1. Decomposition task of initial contraction for sequence of solitons for the Ballmer series. 
calculated solutions of the decomposition task of initial contraction for sequence of soliton solutions (Figure 1).

Detail differential ST simulation allows proposing also internal structures of molecules and atoms in chemical physics, based on electron, proton and neutron particle thermodynamics. By that we follow to $L$. de Broglie's and D. Bohm's methodologies [11]-[13]. This and next sections consider in detail important ST items from elementary particles up to nuclear simulation.

We can derive equations, which describe the distribution of electrical potential and concentration of particles in polarized spaces of electrons, positrons, protons and neutrons [8]. Equation for electrical potential $\varphi$ in polarized ST can be written in form

$$
D^{2} \Delta \varphi=2 \operatorname{sh} \varphi,
$$

where $\varphi$ is related to its characteristic value $\varphi_{0}=T / e ; D^{2}=T / 4 \pi n_{0} e^{2}:$ the Debye radius; $e$ : the value of ultra-elementary electrical charge; $n_{0}$ : characteristic concentration of DM particles (HMBs).

In case of spherical symmetry the following equation can be written

$$
\frac{D^{2}}{r^{2}} \frac{d}{d r}\left(r^{2} \frac{d \varphi}{d r}\right)=2 \operatorname{sh} \varphi .
$$

We shall bring typical solutions of the Equation (12) for polarized electron space (Figure 2). A principal important particularity of distribution presented is potential pit and barrier on external border of polarized space with distribution $\varphi(r)$ break [8]. Induced by electron core electrical charge is concentered at the external boundary. Due to charge symmetry the provided results are also correct for the structure of polarized positron space (in this case the sign of $\varphi$ should be changed).

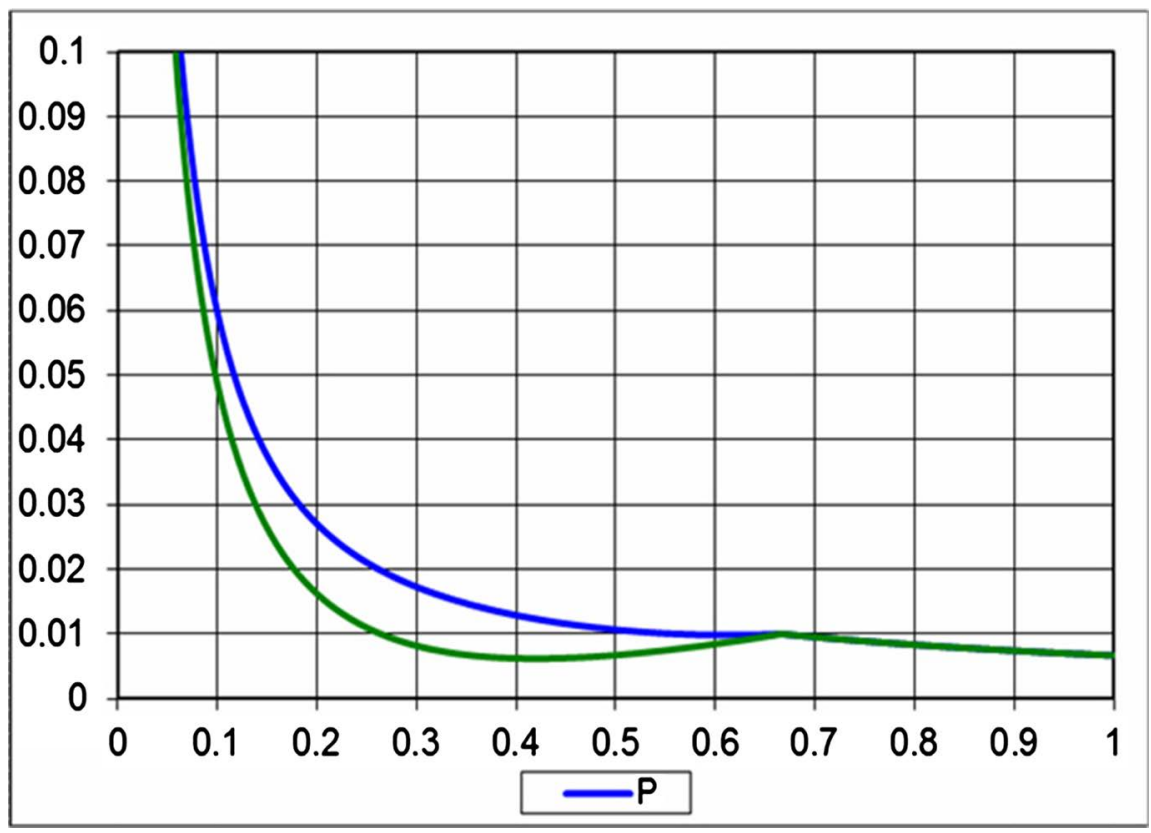

Figure 2. Potential distribution in electron screening space for two $D$. 
The scheme of polarized electron space is shown on Figure 3(a). There are shown the electron core with the radius $r \sim 10-17 \mathrm{~m}$, part of the polarized space (the screening sphere filling HMBs) with the radius $r \sim 5 \times 10^{-11} \mathrm{~m}$.

It is possible to model protons and antiprotons in such way. It is supposed that whole positive charge of proton is situated in its center (Figure 3(b)). The dimension of this center is less than $10^{-17} \mathrm{~m}$. It is surrounded by sphere of polarized "liquid" DM. Dimension of this area is about $0.8 \times 10^{-15} \mathrm{~m}$. It is defined by characteristic size of proton. There is a sphere of "gaseous" DM around the "liquid" one. The modeling of proton bi-layer is possible via integration of Equation (14) for electrical potential with the help of diverse equations of state (for liquid and gaseous phases of DM). The obtained solutions are similar to the electron space solutions [8]. It should be noted that there are two potential holes and two barriers with their radiuses about $r \approx 0.8 \times 10^{-15} \mathrm{~m}$ and $r \approx 5 \times 10^{-11} \mathrm{~m}$. The obtained proton structure is stable. Antiproton structure is similar with proton due to charge symmetry (except for $\varphi$ sign).

Following our methodology we show now shortly the scheme of neutron as a positive nuclear of proton with Debye screening (up to $r_{0}=0.8 \times 10^{-15} \mathrm{~m}$ ) by liquid layer of HMB dipoles and stationary electron, presenting on boundary of Debye region (Figure $3(\mathrm{c})$ ). Decay of neutron gives naturally proton, electron and antineutrino, which presents itself isolated soliton in DM gaseous medium. Exact soliton solutions for neutrino and antineutrino were obtained in [21].

The model of hydrogen atom consists of positive charge in the center $(r<0.4$ $\left.\times 10^{-16} \mathrm{~m}\right)$ and two layers of liquid $\left(0.4 \times 10^{-16}<r<0.8 \times 10^{-15}\right)$ and gaseous $(0.8$ $\times 10^{-15}<r<0.5 \times 10^{-10}$ ) polarized DM space (Figure 4). There is also stationary electron at the external boundary of polarized space $\left(r=0.5 \times 10^{-10} \mathrm{~m}\right)$. This motionless state of electron is achieved by potential distribution in electron and proton models and equilibrium between $\operatorname{grad} \varphi$ and $\operatorname{grad} p$.

The existence of polarized space around atoms with their spherical shape (van der Waals spheres) has been proved by chemistry of crystals and scanning probe microscopy. It is possible to manipulate the separate spheres with needle of tunnel microscope. We can move these spheres or build some figures of them.

\section{Some Technical Applications}

Some basic complexities of designing high temperature air-breathing engines

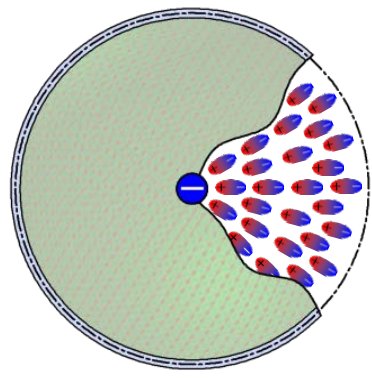

(a)

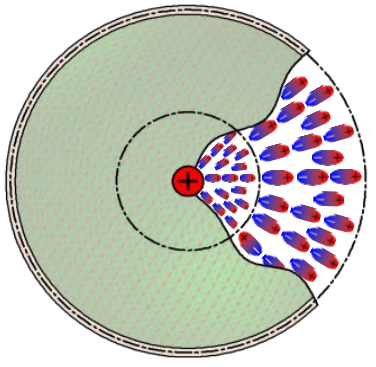

(b)

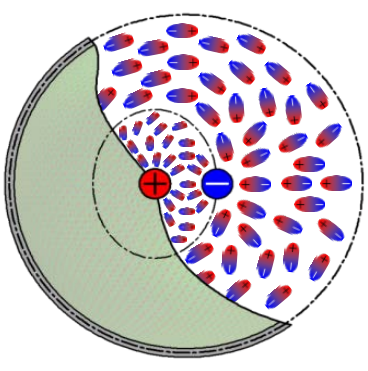

(c)

Figure 3. Scheme of screening spaces of electron (a), proton (b) and neutron (c). 


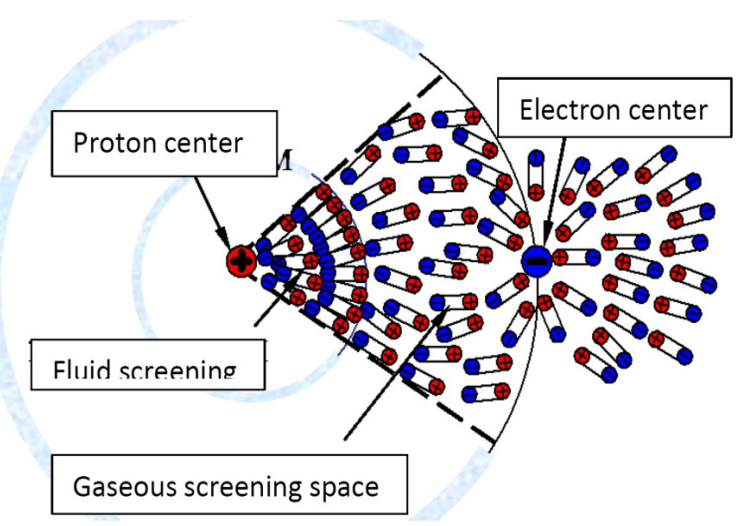

Figure 4. Scheme of hydrogen atom.

relate to origin of quantum effects (so-called "unexpected" heat) of working process. This additional heat essentially complicates coordination of engine basic components (for a turbojet - compressor, combustor and turbine, for a highspeed air-breathing engine - air inlet, isolator, combustor, and nozzle). In our simulations we include into account "unexpected" heat as radiate heat and heat transfer to ST using system (11).

Propulsion combustion chambers with temperatures above $3000 \mathrm{~K}$, scramjet chambers with temperatures near $2500 K$ and other jet engine combustors with temperatures near $2000 \mathrm{~K}$ are strongly influenced by radiate heat transfer. Radiate heat loads depend on temperature's fourth power. Radiate heat transfer simulation usually requires the solution of radiate transfer equation, which depends on special, directional and spectral variables. Examples such types modeling were realized for rocket combustion chambers and have good enough progress. At the same time the practice realization of scramjet propulsion has great difficulties. One of these reasons is a detrimental operation mode called unstart.

Using our theoretical model we would like to explain the similar unstart mode, which don't allow us to realize supersonic burning in scramjet with positive thrust. This operation is the unstart mode, when radiation and shocks (pseudo-shocks system) are destroying supersonic flow in combustor channel.

Figure 5 presents typical results this mode simulation and shows Mach number and pressure counter lines inside channel without burning (a) and with burning (b). In the last mode heat addition destroys supersonic burning and shock wave system is located in the inlet zone. Our simulation based on system (11) is relevant to high-class models based on real 3D geometry of flow path.

The typical examples of investigation of steady and unsteady working modes of the bypass gas turbine engines are shown in Figure 6. We present simulation of whole flow passages for bypass gas turbine engine with afterburning trust 80 $\mathrm{kN}$.

Figure 6 illustrates streamlines and Mach number counter distributions in whole flow passage of multi regime engine with bypass ratio 0.17 and the 3 stages fan, 6 stages high pressure compressor, annular main combustor, one stage 
high and low pressure turbines and afterburner. Additional pressure losses in main combustors demands very accurate determination high pressure turbine cross section and correlation of power for compressor driving. Here we have the decreased flow capacity of the compressor and led finally to increased turbine inlet temperatures.

At the last we present solutions of external reentry shuttle problems. Flow structure and surface temperature distribution for two typical shuttles show on Figure 7. On simulate regime the flight Mach number equals 5 with the angle of attack $5^{\circ}$. These calculations use the equilibrium approach based on (11).

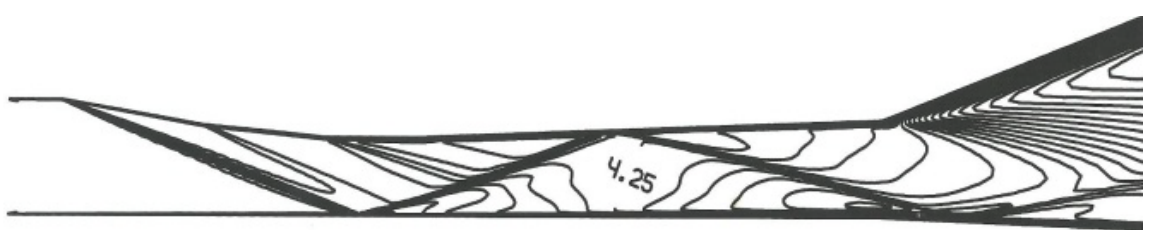

(a)

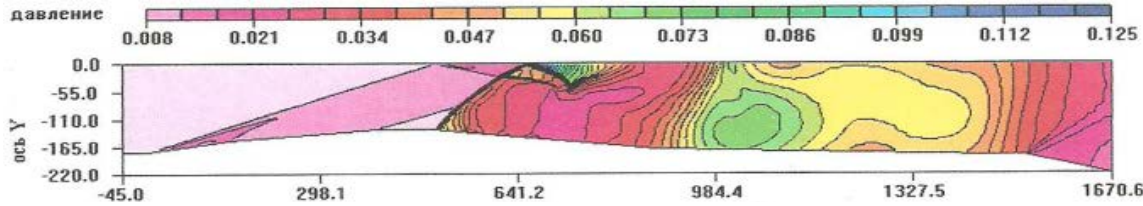

(b)

Figure 5. Mach number lines without burning (a) and pressure counter lines with burning on unstart mode (b) inside high speed channel.

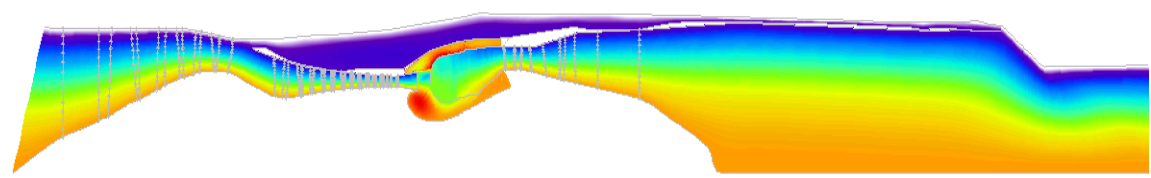

(a)

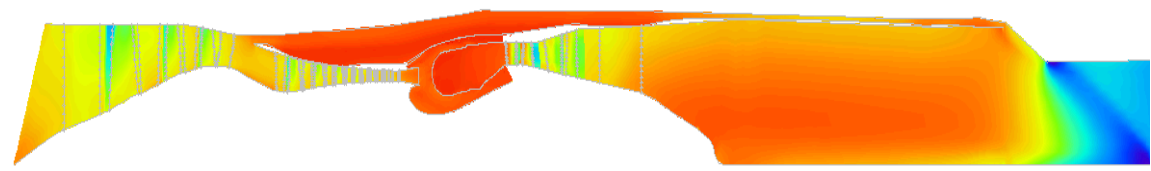

(b)

Figure 6. Streamlines (a) and Mach number (b) counter distributions in whole flow passage of multi regime bypass GTE.

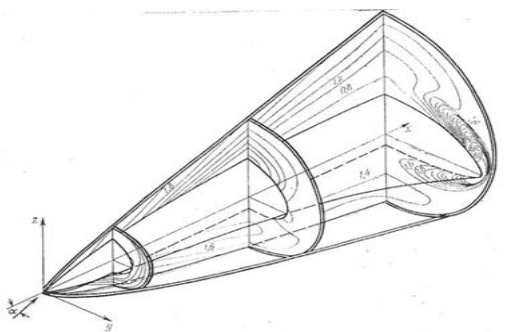

(a)

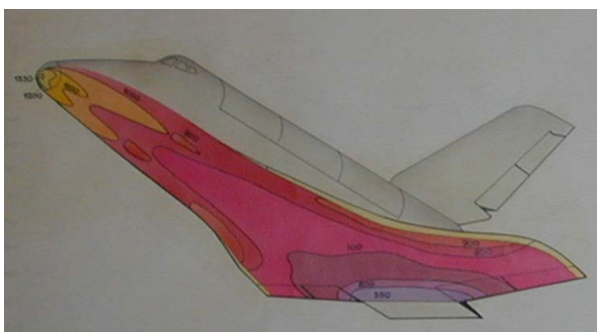

(b)

Figure 7. Flow structure (a) and surface temperature (b) distribution for two typical shuttles. 


\section{Conclusions}

Deep unity of classic and quantum physics at the space thermostat (ST) presence, which fulfilled all space by the temperature $T_{0}=2.73 \mathrm{~K}$, has been demonstrated. Real physics is based on the principle of Galileo's relativity (invariance) and Newton's laws of motion. After linearization procedure we get quantum modern physics. The modern theoretical physics bases on the Lorentz invariance. The Noether theorem, the formalism by Lagrange and Hamilton can't by right in such type cases of opened systems with the ST presence.

\section{References}

[1] Regener, E. (1933) The Energy Flux of Cosmic Rays. Zeitschrift für Physik, 80, 666669. https://doi.org/10.1007/BF01335703

[2] Shmaonov, T.A. (1957) Methodology of Absolute Measurements for Effective Radiation Temperature with Lower Equivalent Temperature. Apparatuses and Technique of Experiment, 1, 83-86.

[3] Penzias, A.A. and Wilson, R.W. (1965) A Measurement of Excess Antenna Temperature at $4080 \mathrm{~m} / \mathrm{s}$. Astrophys. J., 142, 419-421. https://doi.org/10.1086/148307

[4] Smoot, G.F., Gorenstein, M.V. and Muller, R.A. (1977) Detection of Anisotropy of the Cosmic Blackbody Radiation. Phys. Rev. Let., 39, 898-901.

https://doi.org/10.1103/PhysRevLett.39.898

[5] Smooth, G.F. (1977) Anisotropy of Background Radiation. Uspekhi Fizicheskih Nauk, 177, 1294-1318.

[6] Rubin, V. (1998) Dark Matter in the Universe. Scientific American, 1, 106-110.

[7] Moskowitz, G. (2006) Dark Matter Hides, Physicists Seek. Stanford Report.

[8] Ivanov, M.J. (2012) Space Energy. In: Ahmed, A.Z., Ed., Energy Conservation, INTECH, 3-56.

[9] Lax, P.D. (1968) Integrals of nonlinear equations of evolution and solitary waves. Comms. on Pure and Appl. Math., XXI, No. 5, pp.467-490. https://doi.org/10.1002/cpa.3160210503

[10] Madelung, E. (1926) Quantentheorie in Hydrodynamischer Form. Z. Physik, 40, 322-326. https://doi.org/10.1007/BF01400372

[11] Bohm, D. (1952) A Suggested Interpretation of the Quantum Theory in Terms of "Hidden" Variables I. Phys. Rev., 85, 166-179. https://doi.org/10.1103/PhysRev.85.166

[12] Bohm, D. (1952) A Suggested Interpretation of the Quantum Theory in Terms of "Hidden" Variables II. Phys. Rev., 85, 180-193. https://doi.org/10.1103/PhysRev.85.180

[13] Bohm, D. and Vigier, J.P. (1954) Model of the Causal Interpretation of Quantum Theory in Terms of a Fluid with Irregular Fluctuations. Phys. Rev., 96, 208-216. https://doi.org/10.1103/PhysRev.96.208

[14] Gibbs, J.W. (1902) Elementary Principles in Statistical Mechanics, Developed with Especial Reference to the Rational Foundation of Thermodynamics. Yale Univ. Press. https://doi.org/10.5962/bhl.title.32624

[15] Louis de Broglie (1961). Introduction a la theorie des particules. Gauthier-Villars, Paris.

[16] Terletskiy, Y.P. (1994) Statistical Physics, M: High School. 
[17] Noether, E. (1918) Invariant Variation Problems. Math-Phys., 235-257.

[18] Landau, L.D. and Lifshitc, E.M. (1973) Theory of Field. Theor. Phys., 2.

[19] Ovsiannikov, L.V. (1981) Lectures on Gas Dynamics Bases. M.: Nauka.

[20] Mathematical Models of Gas Turbine Engines and Their Components, AGARD Lecture Series TCP 02/LS, 198, 1994.

[21] Ivanov, M.J. and Terentieva, L.V. Exact Solutions of Two-Fluid Approach Equations in Aerospace Plasmadynamics. AIAA Paper No. 2003-0843, 8 p.

Submit or recommend next manuscript to SCIRP and we will provide best service for you:

Accepting pre-submission inquiries through Email, Facebook, LinkedIn, Twitter, etc. A wide selection of journals (inclusive of 9 subjects, more than 200 journals)

Providing 24-hour high-quality service

User-friendly online submission system

Fair and swift peer-review system

Efficient typesetting and proofreading procedure

Display of the result of downloads and visits, as well as the number of cited articles

Maximum dissemination of your research work

Submit your manuscript at: http://papersubmission.scirp.org/

Or contact jamp@scirp.org 